\title{
Deep Learning in Mobile Computing: Architecture, Applications, and Future Challenges
}

\author{
Xiaoxian Yang $\mathbb{D}^{1}{ }^{1}$ Zhiyuan Tan, ${ }^{2}$ and Zhiling Luo $^{3}$ \\ ${ }^{1}$ School of Computer and Information Engineering, Shanghai Polytechnic University, Shanghai, China \\ ${ }^{2}$ School of Computing, Edinburgh Napier University, Edinburgh, Scotland, UK \\ ${ }^{3}$ Alibaba Group, Hangzhou, China \\ Correspondence should be addressed to Xiaoxian Yang; xxyang@sspu.edu.cn
}

Received 19 July 2021; Accepted 19 July 2021; Published 18 August 2021

Copyright ( 2021 Xiaoxian Yang et al. This is an open access article distributed under the Creative Commons Attribution License, which permits unrestricted use, distribution, and reproduction in any medium, provided the original work is properly cited.

With the emergence of big data and efficient computing resources, deep learning has made breakthrough in many areas of artificial intelligence. However, when the training data are increased, the deep learning model may have tens of billions or even hundreds of billions of parameters without any pruning. Mobile networks use a distributed architecture, which means that all computers or devices that are part of it share the workloads in the network. This method can significantly improve the training efficiency of deep learning and further increase its application range. In this special issue, we selected 13 papers. A summary of these papers is outlined as follows.

In the paper entitled "Swarm Differential Privacy for Purpose Driven Data-Information-Knowledge-Wisdom Architecture" by Y. Li et al., they explore DIKW architecture through the applications of the popular swarm intelligence and differential privacy. The proposed approach is demonstrated through the application of personalized data that is based on the open-source IRIS dataset. This experiment demonstrates the efficiency of Swarm Intelligence in reducing computing complexity.

In the paper entitled "Research on Real-Time Anomaly Detection of Fishing Vessels in a Marine Edge Computing Environment" by J. Huang et al., they present a real-time anomaly detection model (RADM) for fishing vessels based on edge computing. The model runs in the edge layer, making full use of the information of moving edge nodes and nearby nodes and combines a historical trajectory extraction detection model with an online anomaly detection model to detect anomalies. Online anomaly detection algorithms detect anomalous behavior based on spatiotemporal neighborhood similarity and reduce the impact of anomaly evolution.

In the paper entitled "The Traffic Flow Prediction Method Using the Incremental Learning-Based CNN-LTSM Model: The Solution of Mobile Application" by Y. Shao et al., they proposed an incremental learning-based CNN-LTSM model, IL-TFNet, for the traffic flow prediction. The lightweight convolution neural network-based model architecture is designed to process spatiotemporal and external environment features simultaneously to improve the prediction performance and prediction efficiency of the model. Experiments have proved that compared with other traffic flow prediction models, the IL-TFNet model performs well in short-term traffic flow prediction.

In the paper entitled "Towards Activity Recognition through Multidimensional Mobile Data Fusion with a Smartphone and Deep Learning" by Cao et al., they use an Android smartphone to collect the data of six basic behaviors of human, which are walking, running, standing, sitting, going upstairs, and going downstairs, through its acceleration sensor and use the classic model of deep learning $\mathrm{CNN}$ (convolutional neural network) to fuse those multidimensional mobile data, using TensorFlow for model training and test evaluation. The generated model is finally transplanted to an Android phone to complete the mobileend activity recognition system.

In the paper entitled "Active Synchronous Control Strategy of Distributed Power Grid Connection Based on Mobile Network" by Zhang et al., they present an active synchronous 
control scheme for distributed power grid connection based on the mobile network. This scheme avoids the disadvantages of the traditional control method, such as long waiting time and low reliability, and can meet the requirements of "the hotplug online" distributed power grid connection. The scheme uses the mobile network to collect and detect voltage slip information between the distributed power system and the large power grid before operation. When the voltage slip cannot meet the requirements of grid connection, the side voltage information of the distributed power system is actively regulated. The amplitude difference and phase difference signals are, respectively, adjusted by PI, and the generated adjustment signals are, respectively, sent to the corresponding controllers through the mobile network with different weight coefficients. After the receiver receives the regulated signal, it changes the amplitude and frequency of its output voltage by shifting the pendent characteristic curve, to realize the distributed power system side voltage and the large power grid side voltage eventually.

In the paper entitled "QoS-Based Multicast Routing in Network Function Virtualization-Enabled Software-Defined Mobile Edge Computing Networks" by Sun et al., they study QoS-guaranteed multicasting routing with network function virtualization (NFV) in MEC. Specifically, data should pass through a service function chain before reaching destinations along a multicast tree with minimal computational cost and meeting QoS requirements. Furthermore, to overcome the problems of traditional IP multicast and software-defined multicasting approaches, they propose an implementable multicast mechanism that delivers data along multicast trees but uses unicast sessions. They finally evaluate the performance of the proposed mechanism based on experimental simulations.

In the paper entitled "Efficient Computation Offloading for Service Workflow of Mobile Applications in Mobile Edge Computing" by Yuan et al., they proposed a blocking- and delay-aware schedule strategy for MEC environment service workflow offloading. First, the workflow was modeled in mobile applications and the buffer queue in servers. Then, the server collaboration area was divided through a collaboration area division method based on clustering. Finally, an improved particle swarm optimization scheduling method was utilized to solve this NP-hard problem. Many simulation results verified the effectiveness of the proposed scheme. This method is superior to existing methods, which effectively reduces the blocking probability and execution delay and ensures the quality of experience of the user.

In the paper entitled "Research on Target Tracking Algorithm Based on Siamese Neural Network" by Pang et al., they study the target tracking algorithm based on a deep Siamese network. Aiming at the situation that the tracking process is not robust, such as drift or missing the target, the tracking accuracy and robustness of the algorithm are improved by improving the feature extraction part and online update part. They add SEblock and temporal attention mechanism (TAM) to the framework of the Siamese neural network. SE-block can refine and extract features; different channels are given different weights according to their importance, which can improve the discrimination of the network and the recognition ability of the tracker. The temporal attention mechanism can update the target state by adjusting the weights of samples in the current frame and historical frame to solve the model drift caused by the existence of the similar background. They use the crossentropy loss to distinguish the targets in different sequences so that their distance in the feature domain is longer and the features are easier to identify.

In the paper entitled "BFLP: An Adaptive Federated Learning Framework for Internet of Vehicles" by Peng et al., they propose a blockchain-based federated learning pool (BFLP) framework. BFLP allows the models to be trained without sharing raw data, and it can choose the most suitable federated learning method according to actual application scenarios. Considering the poor computing power of vehicle systems, they construct a lightweight encryption algorithm called CPC to protect privacy. To verify the proposed framework, they conduct experiments in obstacleavoiding and traffic forecast scenarios. The results show that the proposed framework can effectively protect the user's privacy, and it is more stable and efficient compared with the traditional machine learning technique. In addition, they compare the CPC algorithm with other encryption algorithms. And, the results show that its calculation cost is much lower compared to other symmetric encryption algorithms.

In the paper entitled "Air Quality Prediction Based on a Spatiotemporal Attention Mechanism" by Zou et al., they propose a long short term memory (LSTM) air quality prediction model based on a spatiotemporal attention mechanism (STA-LSTM). The model uses an encoder-decoder structure to model spatiotemporal features. A spatial attention mechanism is introduced in the encoder to capture the relative influence of surrounding sites on the prediction area. A temporal attention mechanism is introduced in the decoder to capture the time dependence of air quality. In addition, for spatial data such as point of interest (POI) and road networks, they use the LINE graph embedding method to obtain a low-dimensional vector representation of spatial data to obtain abundant spatial features. They evaluate STA-LSTM on the Beijing dataset, and the root mean square error (RMSE) and $R$-squared $\left(R^{2}\right)$ indicators are used to compare with six benchmarks. The experimental results show that the model proposed can achieve better performance than the performances of other benchmarks.

In the paper entitled "Hard Disk Drive Failure Prediction for Mobile Edge Computing Based on an LSTM Recurrent Neural Network" by Shen et al., they propose an LSTM recurrent neural network-based HDD failure prediction model, which leverages the long temporal dependence feature of the drive health data to improve the prediction efficiency. In addition, they design a new health degree evaluation method, which stores current health details and deterioration. The comprehensive experiments on two real-world hard drive datasets demonstrate that the proposed approach achieves good prediction accuracy with low overhead.

In the paper entitled "Deep Learning for Mobile Crowdsourcing Techniques, Methods, and Challenges: A Survey" by Liu et al., the complexity of mobile crowdsourcing tasks makes it difficult to find the best solution under the constraints of limited computing resources and various tasks. In the past decades, many researchers have devoted themselves to this hot topic and brought various cutting-edge resolutions. In this case, 
they review the current research status of deep learning for mobile crowdsourcing from the perspectives of techniques, methods, and challenges. Finally, they list a group of remaining challenges that call for an intensive study in future research.

In the paper entitled "Optimization Strategy of Multiarea Interconnected Integrated Energy System Based on Consistency Theory" by Yu et al., they study a collaborative optimization strategy of an integrated energy system aiming at improving the energy efficiency for the cluster optimization of an integrated energy system (IES). They propose a discrete consistency method based on the coordination optimization method. An IES model considering the mixed energy supply of electricity, heat, and gas is constructed in a single region. And, then, an objective function with the maximum return is established, on the premise of assuming that the prices of electricity, heat, and gas can be used as an economical means to adjust the energy utilization. Finally, the consistency theory is applied to the IES, and the improved discrete consistency algorithm is utilized to optimize the objective function.

The Guest Editors would like to express their deep gratitude to all the authors who have made their valuable contributions and to the numerous and highly qualified anonymous reviewers. We think that the selected contributions, which represent the current state of the art in the field, will be of great interest to the community.

\title{
Conflicts of Interest
}

The Guest Editors declare no conflicts of interest.

\author{
Xiaoxian Yang \\ Zhiyuan Tan \\ Zhiling Luo
}

